improvement in median FC levels was seen in those dying from malignancy (1130 to $250 \mathrm{ug} / \mathrm{g}$ in UC and 588 to $490 \mathrm{ug} /$ $\mathrm{g}$ in $\mathrm{CD})$.

When stratified by age of death the FC measurements were similar between age groups.

Conclusions Serious and fatal non-IBD related disease does not seem to lead to spontaneous improvement or deterioration in luminal inflammation prior to death, with no significant 'burn out' of IBD activity seen. For patients approaching the end of their life the aim of treatment focuses on maximising quality of life, but there remains variation in practice around stopping or continuing IBD therapies, and further research in this area is required. This study suggests that for patients with IBD an individualised approach is required, and for many, good palliative care will include active and aggressive management of their IBD.

\section{PMO-14 USE OF NON-INVASIVE SURROGATE MARKERS TO MONITOR VEDOLIZUMAB THERAPY IN INFLAMMATORY BOWEL DISEASE}

${ }^{1}$ John Barragry*, 'Konstantinos Fragkos, 'Greg Sebepos-Rogers, 'Hajeena Saravanapavan, ${ }^{2}$ Hui Fen Koo, ${ }^{2}$ Ho Juen Ko, ${ }^{1}$ Ioanna Parisi, ${ }^{1}$ Edward Seward, 'Sara McCartney, ${ }^{1}$ Stuart Bloom, 'Shameer Mehta, 'Farooq Rahman. 'Dept. Gastroenterology, University College Hospital, London, UK; ${ }^{2}$ University College London Medical School, London, UK

\subsection{6/gutjnl-2021-BSG.153}

Introduction We provide long-term data on the effectiveness of Vedolizumab (VDZ) in the management of inflammatory bowel disease (IBD) by reviewing the response of surrogate markers of disease activity to VDZ therapy, including HarveyBradshaw Index (HBI) for Crohn's disease (CD) and Simple Colitis Clinical Activity Index (SCCAI) for ulcerative colitis (UC), c-reactive protein (CRP), and faecal calprotectin (FCP).

Methods A consecutive cohort of 193 adult IBD patients (90 $\mathrm{CD}$ and 103 UC/IBD-unspecified) who commenced VDZ between May 2015 and June 2019 at a tertiary IBD centre was retrospectively reviewed. Markers of disease at baseline, 1 year $(\mathrm{n}=193), 2$ years $(\mathrm{n}=157)$ and 3 years $(\mathrm{n}=104)$ were assessed. Statistical analyses were performed using SPSS 25.0 , with significance assigned to a p-value of $<0.05$ in bootstrap t-testing.

Results Overall, $77.8 \%$ and $93.2 \%$ of patients with $\mathrm{CD}$ and UC respectively had undergone more than one baseline assessment modality prior to VDZ therapy. At baseline for CD and UC patients respectively; a) median disease activity index (DAI) score was HBI 5 (interquartile range (IQR): 2-8) and SCCAI 5 (IQR: 3-8), b) median CRP was $9.9 \mathrm{mg} / \mathrm{L}$ (IQR: 3.73-22.5 $\mathrm{mg} / \mathrm{L}$ ) and $4.8 \mathrm{mg} / \mathrm{L}$ (IQR: $1.5-10.5 \mathrm{mg} / \mathrm{L}$ ), c) median FCP was $656.5 \mu \mathrm{g} / \mathrm{g}$ (IQR: 301-1472 $\mu \mathrm{g} / \mathrm{g}$ ) and 1190 $\mu \mathrm{g} / \mathrm{g}$ (IQR: $386.5-2639 \mu \mathrm{g} / \mathrm{g}$ ).

Following initiation of VDZ therapy, DAI scores indicated a favourable response in patients with colonic CD disease and all UC disease subtypes from visit 2 onwards compared to baseline $(p<0.05$, Figure $1 A, B)$. There was a significant fall in CRP in colonic but not ileal CD patients $(p<0.05$ at multiple timepoints after visit 4 in Year 1), a trend that persisted during year 1 and extended up to year 3 (Figure 1C). In UC patients, a significant fall was present from visit 3 in Year 1 and persisted in E1 and E2 UC patients compared to baseline $(p<0.05$, Figure 1D). There was a significant reduction in FCP over time in patients with colonic CD and E3 UC $(\mathrm{P}<0.01$, Figure 1E,F).

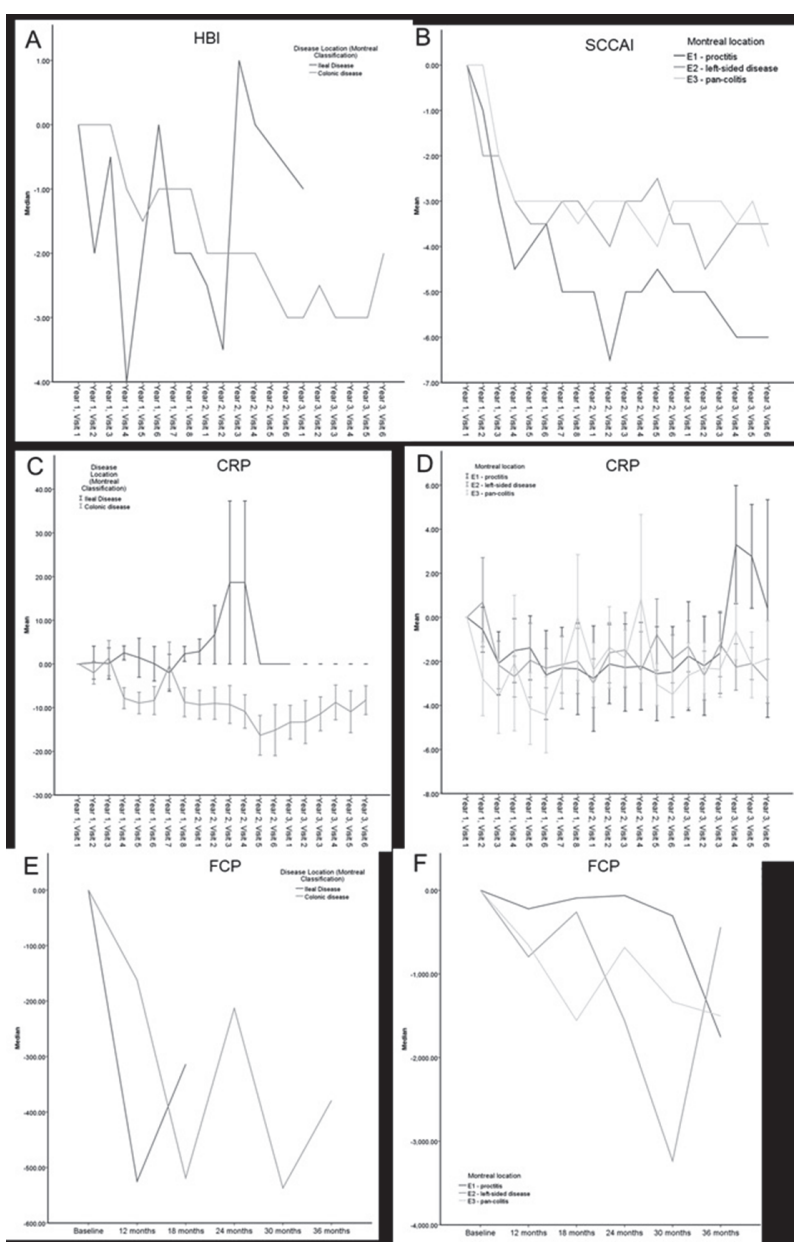

Abstract PM0-14 Figure 1

Conclusion In our single centre retrospective cohort analysis of 193 adult IBD patients, we observed a clinically and statistically significant improvement in markers of disease activity, including DAI scores, CRP and FCP. These surrogate markers allow non-invasive monitoring of VDZ response, particularly in patients with colonic CD and UC.

\section{PMO-15 TRANSITIONING FROM INTRAVENOUS TO SUBCUTANEOUS VEDOLIZUMAB IN PATIENTS WITH INFLAMMATORY BOWEL DISEASE (TRAVELESS)}

${ }^{1}$ Esther Ventress*, 'Sohail Rahmany, 'David Young, 'Marion Bettey, ${ }^{1}$ Clare Harris, ${ }^{2}$ Helen Moyses, ${ }^{1}$ Trevor Smith, ${ }^{1}$ Richard Felwick, ${ }^{1}$ Markus Gwiggner, ${ }^{1,3}$ Fraser Cummings. 'University Hospital Southampton NHS Foundation Trust, Southampton, UK; ${ }^{2}$ NIHR Biomedical Research Unit, Southampton General Hospital, Southampton, UK; ${ }^{3}$ Faculty of Medicine, University of Southampton, Southampton, UK

\subsection{6/gutjnl-2021-BSG.154}

Introduction In May 2020, subcutaneous (SC) vedolizumab was approved for use in Inflammatory Bowel Disease (IBD). Patients with IBD have a number of risk factors for a poor outcome from SARS-CoV-2 infection and managing this risk by reducing hospital visits is crucial. Currently there is no information on the process or outcomes of transitioning patients established on intravenous (IV) vedolizumab to SC.

Methods This is a prospective service evaluation of adult patients who are either stable on IV vedolizumab or have 
been newly started and opted for SC administration. Between October and December 2020, all suitable patients attending our infusion centre for vedolizumab were offered the option to switch to SC. Initially, the aim was to offer a SC dose to patients in place of their IV infusion with injection training by IBD specialists. This proved to be a challenge as it left a narrow window of time for homecare deliveries to be arranged for subsequent doses. Therefore, the remaining patients who agreed to the switch received an IV infusion at their baseline review, with the aim of administering the first SC dose in place of the next scheduled IV dose.

Outcomes include reasons for consenting or declining to switch, patient experience with using SC injections and time saved by not needing to travel to the infusion centre. Data on factors associated with poor outcomes from SARS-CoV-2 infection were collected, including co-morbidities, smoking status, concomitant medication and age.

Clinical baseline data collected as part of routine care included disease activity (modified Harvey-Bradshaw Index or Simple Clinical Colitis Activity Index), biochemical results including C-reactive protein, albumin, haemoglobin and platelet count, faecal calprotectin and quality of life using IBDControl. Trough vedolizumab levels were measured in patients who had had at least 3 IV doses previously. Patients will be reviewed after 12 weeks as part of the switching programme. Results 179 patients were offered the opportunity to change to SC vedolizumab $(54.2 \% \mathrm{CD}, 44.1 \% \mathrm{UC}, 1.7 \% \mathrm{IBDU})$, of which 125 (70\%) (64 (51.2\%) CD, 58 (46.4\%) UC and 3 (2.4\%) IBDU) agreed to the switch. The mean age (SD) was 55 (19.4). 11 patients were new to vedolizumab or reloading. The median time taken by patients (leaving home to returning home) to receive their infusions was 180 minutes (IQR 45 to $360)$.

The main reasons for agreeing to switch were patient preference to manage their treatment at home (70.4\%), concerns about contracting an infection at the infusion centre (15.7\%) and difficulty attending the infusion centre (15.7\%). Reasons for patients declining included not wanting to self-inject (28.3\%), needle phobia (15.2\%), and current instability of symptoms (15.2\%). There have been no major adverse events to date.

Conclusions This is a description of a service evaluation design to monitor outcomes in patients who have consented to transition from IV to SC vedolizumab at one IBD tertiary referral centre.

\section{PMO-16 EFFICACY AND SAFETY OF ELECTIVE SWITCHING FROM INTRAVENOUS TO SUBCUTANEOUS INFLIXIMAB: A MULTI-CENTRE COHORT STUDY}

1Philip J Smith* 1'Daniel Storey, ${ }^{1}$ Belle Gregg, ${ }^{1}$ Lisa Critchley, ${ }^{1}$ June Stenson, ${ }^{1}$ Andrew Kneebone, 'Tracy Rimmer, 'Stevena Burke, ${ }^{2}$ Wan Yi Teoh, ${ }^{2}$ Stephan Vazeille, ${ }^{3}$ Solange Serna, ${ }^{1}$ Ashley Bond, ${ }^{1}$ Alan Steel, ${ }^{1}$ Martyn Dibb, ${ }^{1}$ Paul Collins, ${ }^{1}$ Edmund Derbyshire, ${ }^{1}$ Keith Bodger, ${ }^{1}$ Christopher Probert, ${ }^{3}$ Ajay Verma, ${ }^{1}$ Sreedhar Subramanian. ${ }^{1}$ Department of Gastroenterology, Liverpool University Hospitals Foundation NHS Trust, Liverpool, UK, Liverpool, UK; ' ${ }^{2}$ Liverpool School of Medicine, University of Liverpool, Liverpool, UK; ${ }^{3}$ Department of Gastroenterology, Kettering General Hospital, Kettering, UK

\subsection{6/gutjnl-2021-BSG.155}

Introduction Intravenous (IV) infliximab is a well-established therapy for inflammatory bowel diseases (IBD) patients. A subcutaneous (SC) formulation of infliximab has recently been shown to be as effective as IV infliximab in a randomised trial but there are no real world data to support elective switching. We aimed to assess the effectiveness of an elective switching program from IV to SC infliximab.

Methods Patients on maintenance IV infliximab were included in this retrospective multi-centre cohort study across two sites. Disease activity was monitored serially with Harvey-Bradshaw Index (HBI) for Crohn's disease (CD) and simple clinical colitis activity index (SCCAI) for Ulcerative colitis (UC) at baseline, 3 and 6 months. Faecal calprotectin (FCP), C-reactive protein (CRP) and infliximab levels were recorded at the same time points. Patients on $5 \mathrm{mg} / \mathrm{kg}$ every 8 weeks were switched to SC infliximab $120 \mathrm{mg}$ every other week (EOW), followed by those on 6 and 4 weekly IV infliximab to either EOW or weekly SC infliximab. Inclusion and exclusion criteria for switching were agreed within a standard operating procedure. Data was analysed using SPSS for Windows and Microsoft Excel, were $\mathrm{p}<0.05$ being significant (ns, non-significant).

Results 172 patients (109 CD, 57 UC, 6 IBD-U) were switched to SC infliximab since April 2020 (97 male vs. 75 female; mean age 39.7 years; mean weight $78.6 \mathrm{~kg}) .57 .6 \%$ of patients $(n=99)$ were on concurrent immunomodulators (mean thioguanine level $=259 \mathrm{pmol} / 8 \times 10^{8} \mathrm{RBC}$ ). 152 patients were switched to EOW dosing, 20 to weekly dosing. From baseline to 6 months post switch, HBI, SCCAI, FCP and CRP remained stable ( $p>0.05$, ns), but infliximab levels significantly increased from baseline (baseline mean $=9.8 \mathrm{ug} / \mathrm{mL}$ vs. 3 month mean $=14.6 \mathrm{ug} / \mathrm{mL} ; \mathrm{p}<0.0001)$ and then levels remained high between 3 and 6 month follow up (6 month mean $=15.0 \mathrm{ug} / \mathrm{mL} ; \mathrm{p}>0.05$, ns). 8 patients $(4.65 \%)$ had selflimiting skin injection site reactions, 1 UC patient $(0.58 \%)$ required corticosteroids for a disease flare, and 2 patients $(1.16 \%)$ developed perianal symptoms requiring examination under anaesthesia (EUA). Overall 4 patients (2.36\%) discontinued SC treatment due to adverse events (2 perianal disease patients requiring EUA, 1 flaring UC patient requiring oral steroids and 1 patient with neurological symptoms) and 1 $(0.58 \%)$ planned discontinuation for a patient in the $3 \mathrm{rd}$ trimester of pregnancy.

Conclusions SC infliximab is effective at maintaining remission in IBD patients switched from IV to SC infliximab with no evidence of inferiority at 6 month follow up. There is a significant increase in infliximab levels from baseline to 3 months which is maintained to 6 months post switch. SC infliximab appears to be safe with low rates of injection site reaction or other serious adverse events.

\section{PMO-17 CLINICAL PREDICTORS OF VEDOLIZUMAB RESPONSE IN CROHN'S DISEASE}

${ }^{1}$ Hajeena Saravanapavan*, ${ }^{1}$ Gregory Sebepos-Rogers, ${ }^{1}$ Konstantinos Fragkos ${ }^{1}$ John Barragry, ${ }^{2}$ Hui Fen Koo, ${ }^{2} \mathrm{Ho}$ Juen Ko, ${ }^{1}$ loanna Parisi, ${ }^{1}$ Edward Seward, ${ }^{1}$ Sara McCartney, ${ }^{1}$ Stuart Bloom, ${ }^{1}$ Shameer Mehta, ${ }^{1}$ Farooq Rahman. ${ }^{1}$ Dept. Gastroenterology, University College Hospital, London, UK; ${ }^{2}$ University College London Medical School, London, UK

\subsection{6/gutjnl-2021-BSG.156}

Introduction Vedolizumab (VDZ) is an $\alpha 4 \beta 7$ integrin antagonist licensed for treatment of moderate to severe Crohn's disease (CD). We provide long-term data on best predictors of clinical and corticosteroid-free response, remission and persistence from a tertiary IBD centre. 